\title{
Computer-augmented decision making
}

\author{
C. P. WHALEY \\ Bell-Northern Research, Ottawa, Ontario, Canada
}

\begin{abstract}
The principal judgmental components of multiattribute decision making are examined here with specific reference to how these components can be captured electronically. Once captured, a function, rule, or algorithm may be executed for the integration of this information and the selection of the optimal alternative(s). Two kinds of algorithms are discussed: one based on linear models, the other on fuzzy-set theory and ratio scaling. With on-line support and certain assumptions about human biases (which lead to nonoptimal decisions), the quality of decisions can be enhanced considerably. The principal concerns are with end-user acceptance of computeraugmented decisions.
\end{abstract}

A major premise of the present paper is that what is currently known about the psychology of decision making can be utilized in the design and implementation of decision aids. As of 1975 , there have been more than 100 books, more than 1,300 articles, and more than 800 other publications on behavioral decision theory (Slovic, Fischoff, \& Lichtenstein, 1977). No attempt will be made to review this literature here. Rather, the prospects for computer-augmentation models for multiattribute or multicriteria decision making (Zeleny, 1976) will be considered.

There are three factors that suggest that there is a place for computer-aided decision systems. The first has been mentioned. (1) We do know a great deal about the psychology of unaided human decision making. (2) The current mathematical and computer-oriented approaches to decision making processes have been developed, for the most part, independently of everything known (by psychologists in the area) about the behavioral issues involved in the decision process. (3) Finally, from what is known about human decision theory and from what is known to exist by way of computerized approaches, it appears likely that very powerful decision augmentation aids can be implemented on very small computers with on-line support to their users.

There has already been a groundswell of specialized microcomputers that meet various human needs and wants. There are small and even hand-held devices that, among other things: perform complex calculations (i.e., hand-held calculators); teach children to speak, spell, and do simple arithmetic (e.g., Texas Instruments' Speak \& Spell); teach us other languages (e.g., Craig M100); provide us with music to wake up to, to work to, or to play (e.g., Casio's Melody Alarm watch and Casiotone M-10); allow the deaf to communicate by telephone (e.g., Northern Telecom's Visual Ear); and, permit truly portable computing capabilities (e.g., Radio Shack's TRS-80 pocket computer, or its other hand-held rivals, Sinclair ZX80, Quasar HHC, Panasonic RL-H1000, and Sharp PC-1211). Judging from the proliferation of these devices, it must be assumed that the users are much more accepting of this kind of technology than they typically are of large computers capable of performing the same feats.

The memory requirements for all of these machines are quite small compared with mini- or larger computers; yet the processes that are executed are often very sophisticated and fast.

Given that the preceding facts and trends continue to hold true, there is clearly a place for microcomputerbased decision augmentation. The remainder of this paper describes the multiattribute decision process and how a user interface can be devised to carry out fairly sophisticated decision analyses.

\section{MULTIATTRIBUTE DECISION MAKING}

Multiattribute decision making can be expressed most clearly in matrix form (as in Table 1).

Table 1

Matrix Representation of Multiattribute Decision Making

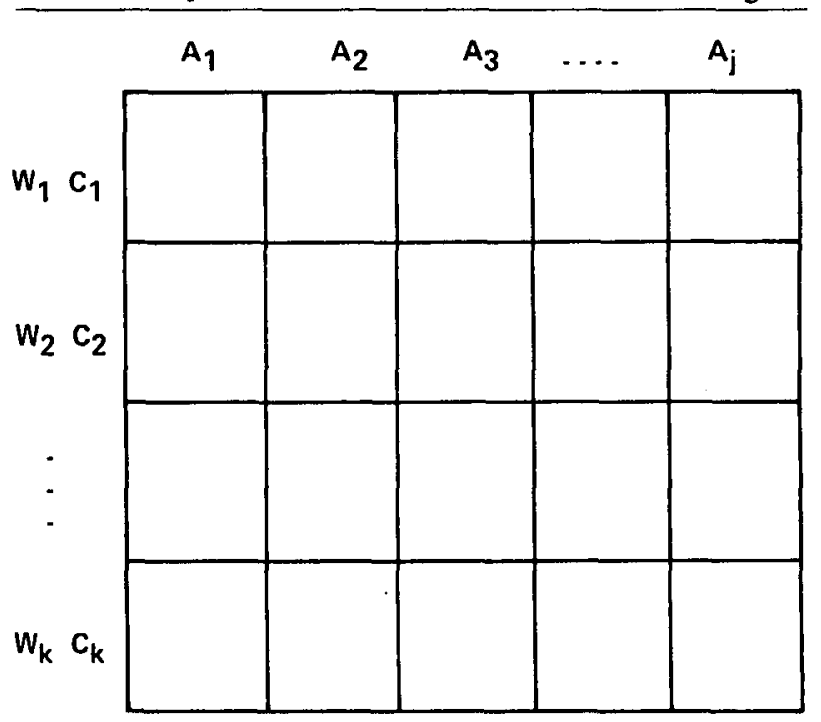


The As along the columns represent different solutions to a problem or different alternatives among which one wishes to choose. The Cs next to the rows of the matrix signify the criteria or attributes that play a role in the decision. When purchasing a home computer, for example, one might consider graphics, the amount of user-programmable memory, reliability, cost, and physical size. An optimal decision requires an evaluation of the alternatives with respect to each criterion (i.e., values assigned to each cell of the matrix).

The Ws next to the Cs are weights to be applied to the criteria. In the home computer example, if cost is more important to the buyer than the graphics capabilities, then cost should receive a greater weight in deciding among the alternatives.

The final but probably most important ingredient is an algorithm, rule, or mathematic function that takes the entries of the matrix plus the weights and generates a single vector of decision values, one for each alternative. The optimal alternative is naturally the one that falls at the appropriate end of this continuum.

\section{Altemative Models}

The principal difference among decision models, then, is in the assignment of weights and in the reduction of the weighted matrix to a vector of decision values.

Linear decision making. Linear decision models may be thought of as a subjective form of multipleregression analysis. That is, the decision vector is determined by simply summing the products of the weights and the ratings across the various criteria for each alternative.

Meehl's (1954) classic book on clinical vs. statistical prediction shows that linear models consistently outperform clinical intuition across a wide variety of tasks. Dawes (Dawes \& Corrigan, 1974; Dawes, Note 1) has subsequently shown that even when the weights are obtained in a nonoptimal manner (e.g., all set to be equal), the results are more accurate than are those determined by clinical judgment or intuition.

Fuzzy decision making. To demonstrate the superiority of linear models over unaided intuition, however, is not to say that linear models are better than models that employ some other algorithm to assign weights and produce the decision vector. Saaty (1977) has promoted a ratio scaling approach for determining weights that permits a simple paired comparison procedure to be used. Yager (1977) has combined Saaty's method for weighting the criteria with a fuzzy-set approach to the decision process itself. He argues that since fuzzy-set theory (Zadeh, 1965, 1968, 1972) and its associated mathematics are specifically oriented to the case in which category membership is unclear, it is ideally suited to the decision process.

The details of fuzzy-set theory or Saaty's (1977) ratio scaling methodology are outside of the scope of this paper, but is worth mentioning that a model of this kind (when implemented as an on-line interactive computer program) provides a very simple user interface, even though the (unseen) mathematics are quite complex (Whaley, 1979).

\section{An Example}

An example of how the multiattribute decision making process can be partitioned to allow easy on-line data collection without losing the power of the algorithm that generates the optimal decision(s) is shown in the next sequence of tables. The example is essentially the same as the one that accompanied the publication of a BASIC program for fuzzy decision making (Whaley, 1979). ${ }^{2}$

Tables 2.5 represent (in abbreviated form) the process by which the user obtains a decision based on his/her own judgments plus an (unseen) algorithm that applies fuzzy-set-theoretic logic and ratio scaling to assign weights and select the best alternative(s). It will become apparent through the example that the process of obtaining the basic data for a linear model approach or many other approaches would be very similar from the user's point of view.

The example is one of choosing among four alternative home computers for purchase. The alternatives are referred to here by the letters A-D. Having selected both a reasonable set of alternatives to choose from and a set of criteria thought to be most appropriate to the decision, the user is ready to begin.

In an iterative fashion, the program prompts the user for the necessary data. Using each criterion in turn, the user assigns a rating to each alternative. It is convenient to use values between zero and one to express the extent to which each alternative possesses each property. Within the fuzzy-set framework, the actual endpoints, zero and one, are not used, since these values would imply that the decision is not fuzzy along at least one of the criteria and, consequently, that the best (or worst) choice is obvious.

A summary of the collective ratings from this first stage is shown in Table 2.

Once the judgments along each of the independent criteria are assigned, the second procedure is begun, that of assigning weights to the criteria. In the spirit of Saaty (1977) and Yager (1977), this is accomplished in the fuzzy decision model by using paired comparison judgments with a rating scale. Typically, a rating scale

Table 2

Ratings of Four Computers

\begin{tabular}{lllll} 
& A & B & C & D \\
\hline Graphics & .4 & .3 & .3 & .8 \\
Cost & .3 & .8 & .5 & .5 \\
Reliability & .7 & .7 & .4 & .3 \\
Memory & .3 & .2 & .7 & .5 \\
Size & .9 & .6 & .7 & .5 \\
\hline
\end{tabular}


Table 3

Rating Scale for Paired Comparison Judgments of the Criteria

\begin{tabular}{cl}
\hline $\begin{array}{c}\text { Degree of } \\
\text { Importance }\end{array}$ & Definition \\
\hline 1 & Equal importance \\
2 & \\
3 & Weak importance of one over the other \\
4 & \\
5 & Strong importance of one over the other \\
6 & \\
7. & Demonstrated importance of one over the other \\
8 & \\
9 & Absolute importance of one over the other \\
\hline
\end{tabular}

Table 4

Ratings of the Criteria

\begin{tabular}{lcc}
\hline \multicolumn{1}{c}{ Pair Presented } & Choice & Rating \\
\hline Graphics-Cost & 2 & 3 \\
Graphics-Reliability & 2 & 7 \\
Cost-Reliability & 2 & 5 \\
Graphics-Memory & 2 & 4 \\
Cost-Memory & 2 & 2 \\
Reliability-Memory & 1 & 2 \\
Graphics-Size & 2 & 3 \\
Cost-Size & 1 & 3 \\
Reliability-Size & 1 & 6 \\
Memory-Size & 1 & 4 \\
\hline
\end{tabular}

Table 5

Final Decision Values

\begin{tabular}{cccc}
\hline $\mathrm{A}$ & $\mathrm{B}$ & $\mathrm{C}$ & $\mathrm{D}$ \\
\hline .23 & .14 & .11 & .06 \\
\hline
\end{tabular}

such as the one in Table 3 is used. The scale is very similar to the one used by Saaty (1977) in his studies. For each pair of criteria, the user not only chooses which one he feels is more important but also indicates how much more important with a value from the rating scale. A sample rating sequence is shown in Table 4.

The remainder of the exercise is carried out by the computer. The weights are determined from the paired comparison judgments, using Saaty's (1977) method, and are applied to the initial table of ratings. Then, using fuzzy logic, the weighted data table is reduced to a vector of decision values, one for each alternative. The largest value is associated with the best choice.

In Table 5 the decision values for the data in Tables 2 and 4 are shown. There is a considerable spread in the values obtained, and Alternative A clearly appears superior. It is of interest to note that had a linear model been used with equal (unit) weights assigned to each criterion, all of the decision values would have been precisely equal. This underscores the importance of both weighting the criteria used and choosing the most appropriate algorithm.

\section{HUMAN DECISION VS. COMPUTER- AUGMENTED DECISION}

It has been demonstrated that unassisted decisions are prone to at least six different kinds of judgmental bias over and above random error (Slovic, in press). It is also apparent that since many of these biases are systematic, there are specifiable rules and operating procedures that can (theoretically) counteract these precursors to nonoptimal decisions (Tversky \& Kahneman, 1974).

Some of these biases can be overcome by simply applying a transformation to the user-provided data that reverses the trend and extent of the bias. Other biases can at least be minimized by partitioning the overall decision task into subtasks that are manageable without bias. This is the approach that has been taken in the example already discussed. Further biases can be reduced by appealing to more than one decision maker. Social decision making theory will not be discussed here, except to say that in some contexts (e.g., committeebased decisions), a multiple-user multiattribute decision analysis approach shows a great deal of promise.

\section{User Acceptance of Computer-Augmented Decisions}

The ultimate question is one that hinges on user acceptance of these heuristics, especially when they are incorporated in small on-line computers. Everyone is undoubtedly familiar with the stereotypic mistrust of computers that pervades our society and the equal and opposite trust in personal intuition. Both of these forces speak against computer augmentation of decision making. On the other hand, most people can also relate to at least a few prominent and frequently expensive bad decisions that they have made.

The area of the acceptability of computer assistance in the human decision process is one that has not received a great deal of attention. It is well established that many people have an (often justified) resistance to computers and computer terminals of any sort. What must be coped with in addition to this is the user's perception of whether there is a close enough match between the decision values provided by the algorithm and his/her own intuitions of what they should be.

It is already established that decisions based on clinical intuition do not correlate well with those obtained through the systematic application of a (linear) decision model. In the original experiments, however, the "intuitive" data and the "linear model" data were collected at different points in time or were obtained from different groups of individuals. If the individual is an active participant in providing the inputs from which the machine determines the decision vector: he or she may perceive a closer correspondence between intuition and computer output than would otherwise be possible. This is an optimistic scenario that has yet to be proved with hard data. 


\section{The Need for an Empirical Approach}

Systems already exist that are not based on behavioral theory or data. Simmons (1979), for example, is marketing a system for computer-assisted social decision making. Consensor, as it is called, does little more than tabulate votes and present the tallies in the form of a bar graph on a color television set. There is nothing wrong with this approach in principle, but it falls so short of what is possible given our current understanding of the field that it is staggering. In a practical sense, Consensor requires that all decisions regardless of complexity be made along a single criterion. It has no builtin mechanism whatsoever for the multiattribute decision process. It should be the responsibility of psychologists (specializing in this area) not only to determine which algorithms offer the best compromise between decision optimality and user acceptance, but, in addition, to expose and discourage the use of systems that fail to meet certain minimal standards of expected utility.

\section{SUMMARY}

This paper has drawn attention to the following issues: (1) People are generally poor decision makers. (2) Computer-assisted decision making, when presented in the form of a stepwise interactive computer program, simplifies the decision process for the decision maker. This simplification can reduce some (although not all) biases inherent in unaided decision making. (3) The state-of-the-art technology permits the implementation of such systems on very small (portable) microcomputers. (4) There is widespread acceptance of other kinds of microprocessor-based devices that satisfy human needs and wants.

It remains to be seen how these basic facts can be drawn upon to overcome the user-acceptance barrier in the domain of computer-augmented decision making.

\section{REFERENCE NOTE}

1. Dawes, R. M. The robust beauty of improper linear models in decision making. Paper presented at the annual meeting of the American Psychological Association, San Francisco, August 1977.

\section{REFERENCES}

Dawes, R. M., \& Corrigan, B. Linear models in decision making. Psychological Bulletin, 1974, 81, 95-106.

МЕEнL, P. E. Clinical versus statistical prediction: $A$ theoretical analysis and review of the literature. Minneapolis: University of Minnesota Press, 1954.

SAATY, T. L. A scaling method for priorities in hierarchical structures. Journal of Mathematical Psychology, 1977, 15, 234281.

Simmons, W. The Consensor: A new tool for decision-makers. Futurist, April 1979, pp. 91-94.

Slovic, P. Toward understanding and improving decisions. In W. C. Howell E. A. Fleishman (Eds.), Human performance and productivity (Vol. 2): Information processing and decision making. Hillsdale, N.J: Erlbaum, in press.

Slovic, P., Fischioff, B., \& Lichtenstein, S. Behavioral decision theory. Annual Review of Psychology, 1977, 28, 1-39.

TVErskY, A., \& KaHneman, D. Judgement under uncertainty: Heuristics and biases. Science, 1974, 185, 1124-1131.

Whaley, C. P. Fuzzy decision making. Interface Age, 1979, 4, 87-91.

Y AGER, R. R. Multiple objective decision-making using fuzzy sets. International Journal of Man-Machine Studies, 1977, 9, 375-382.

ZADEH, L. A. Fuzzy sets. Information and Control, 1965, 8, 338-353.

ZADEH, L. A. Fuzzy algorithms. Information and Control, 1968, 12, 94-102.

ZADEH, L. A. A fuzzy-set theoretic interpretation of linguistic hedges. Journal of Cybernetics, 1972, 2, 4-34.

ZELENY, M. (Ed.). Multiple criteria decision making. Berlin; Springer-Verlag, 1976.

\section{NOTES}

1. They need not be applied in the multiplicative fashion implied by their representation in the table. The fuzzy decision model discussed below uses an exponential weighting function.

2. A FORTRAN version is also available from the author on request. 